\title{
Effect of negative oxygen ions on the characteristics of plasma in a cylindrical DC discharge
}

\author{
Davoud Dorranian · Mahsa Alizadeh
}

Received: 4 January 2014/ Accepted: 5 March 2014/Published online: 3 April 2014

(c) The Author(s) 2014. This article is published with open access at Springerlink.com

\begin{abstract}
Langmuir probe measurements are performed in cylindrical de glow discharge plasma. Plasma was generated in an evacuated glass tube, with circular plane disk electrodes. Measurements were carried out at different points along the axis of tube for different working pressures of pure argon and mixture of argon-oxygen gasses to obtain the plasma density and temperature as well as plasma and floating potentials. Variation of discharge potential as a function of discharge pressure for both plasmas is observed. It is shown that electron temperature, plasma potential, and floating potential in constant current mode and constant pressure are increased from cathode to anode on the axial points of the discharge tube, while electron density is decreased. To sustain the discharge process after adding oxygen to plasma, higher voltage is required since electrons are more energetic at lower density.
\end{abstract}

Keywords Glow discharge plasma - Langmuir probe . Electron density $\cdot$ Floating potential $\cdot$ Electronegative

\section{Introduction}

Classical low pressure electrical discharge has been the topic of several researches in the field of plasma physics for many years. Recently development of plasma technology in industry has motivated plasma physicist to focus their researches on different phenomena occurred in the electrical discharge tube at a wide variety of discharge

D. Dorranian · M. Alizadeh $(\bowtie)$

Plasma Physics Research Center, Science and Research Branch, Islamic Azad University, Tehran, Iran

e-mail: mahsa_sonic@yahoo.com conditions. Thin film deposition, surface etching of materials, and plasma sterilization are some important samples of application of plasma in industry.

The glow discharge is the best known type of nonthermal discharge. A non-thermal glow discharge occurs at low pressures. An electric discharge can be viewed when two electrodes are inserted to a glass tube and connected to a power supply. The tube can be filled with various gasses or evacuated. As the applied voltage across the two electrodes increases, the current suddenly increases sharply at a certain voltage required for sufficiently intensive electron avalanches. If the pressure is low, on the order of a few torrs, and the external circuit has a large resistance to prohibit a large current, a glow discharge develops. This is a low-current, high-voltage discharge widely used to generate non-thermal plasma [1].

In the case of cold dc plasma with Maxwellian distribution, Langmuir probe (LP) is a very useful and trustable convenient device for measuring the local plasma parameter. Langmuir probe is simply a wire immersed in the plasma to determine the electron temperature, electron density, and plasma potential.

In this work, the effect of pressure of the discharge tube on the characteristics of argon and argon-oxygen plasmas is studied experimentally. Measurements were carried out in pure argon and $\mathrm{Ar} /\left(\mathrm{Ar}+\mathrm{O}_{2}\right)=80 \%$ plasmas when the plasma current was kept constant. Oxygen is an electronegative gas. The electronegative gas in the discharge has a strong effect on the electron dynamics behavior, because of the attachment of electrons to the electronegative radicals, thus becoming negative ions. The presence of negative ions complicates the transport properties of the discharge. There has been considerable scientific and technological interest in electronegative plasmas [2-4] and there have been various methods in the determination of negative ion density 


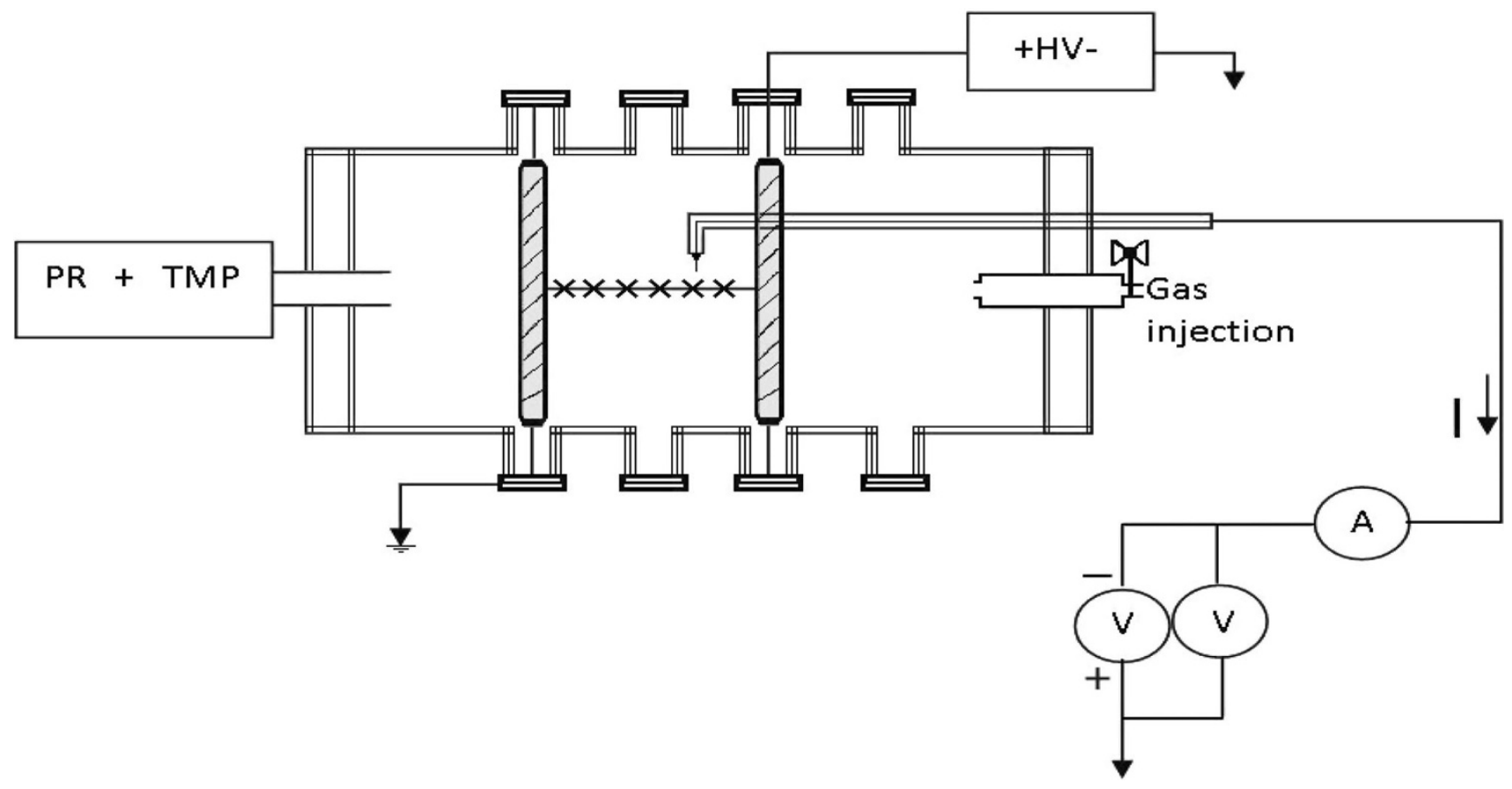

Fig. 1 Schematic view of discharge tube with LP circuit

[2-6]. Electrostatic probes [2-8], laser photodetachment (LPD) [2-9], and laser Thomson scattering (LTS) [2-10] are frequently used diagnostic tools for detecting negative ions in plasma. Among them electrostatic probe technique is simple and inexpensive, and provides the spatial resolution of plasma parameters.

This manuscript is organized as follows: following the introduction, in the second section, we present the experimental setup. Third section is devoted to the results and discussion and the final section includes conclusion.

\section{Experimental setup}

We attempt to experimentally investigate the influence of pressure and working gas on plasma characteristics via the LP technique. The experimental setup consists of an evacuated glass tube illustrated in Fig. 1 with circular disk electrodes at $22 \mathrm{~cm}$ distance from each other used as cathode which is connected to a high-voltage dc power supply and anode which is grounded. The whole tube is evacuated down to $6.2 \times 10^{-5}$ mbar by a turbo molecular pump backed by a rotary pump. During the first experiment argon was used as the working gas and for the second experiment $\mathrm{Ar} / \mathrm{O}_{2}(20 \%)$ gas was introduced into the system under continuous pumping conditions at a working pressures of $1 \times 10^{-2}, 1.9 \times 10^{-2}, 3 \times 10^{-2}$, $4 \times 10^{-2}$, and $5.4 \times 10^{-2}$ mbar. The experiments are performed at constant current mode equal to $10 \mathrm{~mA}$. In fact at each pressure of the discharge tube, we let selfsustaining voltage discharge change such that the discharge current remains constant. Discharge voltage was measured by a high-voltage probe connected between cathode and earth. Plasma current was obtained by measuring the potential difference of a $10 \mathrm{k} \Omega$ resistance which was between the high-voltage power supply and cathode. In this structure, the plasma can be assumed to be cylindrically symmetric.

To determine the plasma parameters, a movable single LP is designed and constructed. The Langmuir probe is made of a narrow quartz pipe with cylindrical tungsten tip. The length of the probe tip is vertically $10 \mathrm{~mm}$ and its radius is $0.3 \mathrm{~mm}$. The probe can be moved longitudinally along the tube axis $(z$ axis). The probe was connected through an electrical circuit to a dc power supply ranging from -50 to $50 \mathrm{~V}$. The probe current as a function of the probe voltage was measured by a convenient digital ampere meter between the probe tip and the ground as shown in Fig. 1.

To measure the plasma parameters the probe was positioned at 6 axial points, which are indicated in Fig. 1. The point $z=0$ is the location of anode and $z=1$ is the first point near anode at which the measurements were started. The Plasma parameters (electron density, electron temperature, plasma potential) were obtained by analyzing the current-voltage characteristic curve of the probe. 


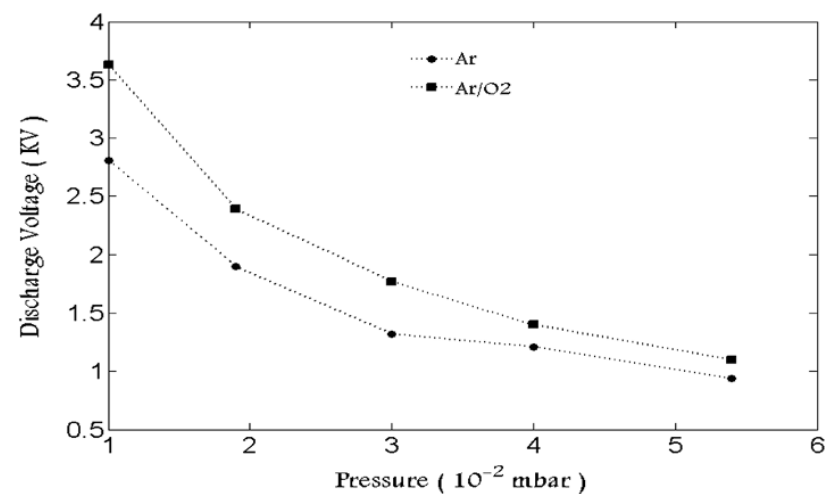

Fig. 2 Glow discharge voltage versus pressure of $\mathrm{Ar}$ and $\mathrm{Ar} / \mathrm{O}_{2}$ in the tube

\section{Results and discussion}

The discharge voltage as a function of pressure for two cases of argon and argon-oxygen plasma is presented in Fig. 2. To have a constant current between electrodes by increasing the pressure of discharge tube the discharge voltage is decreased. In other words, plasma conductivity is increased with increase in the pressure. In plasma, the electrical current is carried by both ions and electrons. For a Lorentzian plasma, in which the accelerated electrons toward anode collide repeatedly with the neutral background gas, the electrical conductivity $\sigma$ is given by microscopic parameters of plasma [11]:

$\sigma=\frac{e^{2} n_{\mathrm{e}}}{m_{\mathrm{e}} v}$

where $m$ and $e$ are the electron mass and electric charge, respectively, $n_{\mathrm{e}}$ is the plasma electron density and $v$ is the collision frequency between plasma electrons and neutral particles. By increasing the working pressure in the discharge tube the electron density is increased. This result is in good agreement with the presented results in Refs. [12-14]. This is also observed from the probe measurements which will be shown later in this manuscript. Of course increasing the pressure will increase the collision frequency. But it can be observed that in this regime of electrical discharge increasing the plasma electron density is the predominated process which leads to increasing the plasma conductivity. Oxygen is an electronegative gas. Adding oxygen to the working gas leads to formation of negative ions in the plasma and decreasing the plasma electron density. Same results have been observed by Yasserian et al. and Noguchi et al. [12, 13, 15]. In this case conductivity of argon-oxygen plasma is smaller than pure argon plasma so a larger discharge voltage is required to sustain the plasma in the tube. This behavior can also be explained by Paschen's law. Paschen's law is an equation (a)

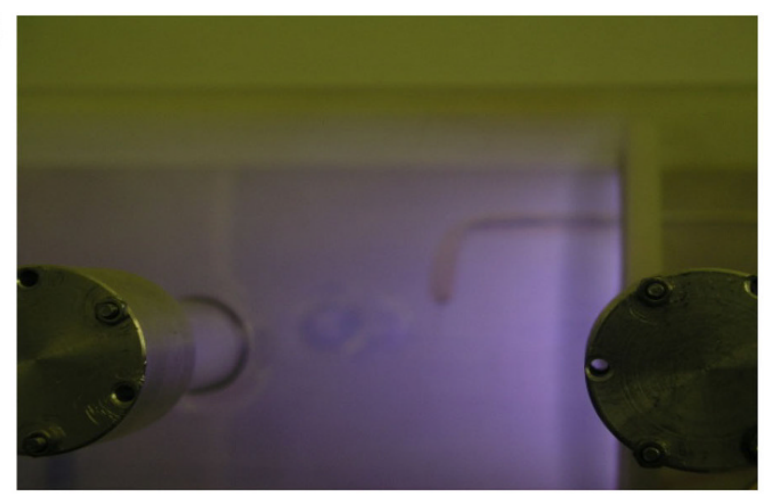

(b)

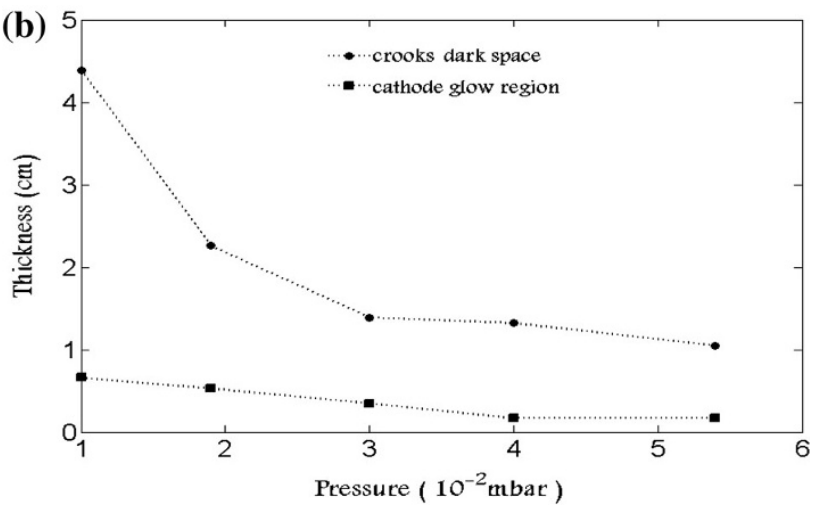

Fig. 3 Ar plasma in the discharge tube (a) and thickness of Crookes dark space and cathode glow regions at different pressures (b)

that gives the breakdown voltage, i.e., the voltage necessary to start a discharge or electric arc, between two electrodes in a gas as a function of pressure and electrodes distance [12]. According to Paschen's law, the voltage necessary to arc across the gap between electrodes decreases as the pressure increases and then increases gradually with the pressure. In Fig. 2, the discharge in plasma tube is in the left hand side of Paschen's curve.

Light emission of the discharge in argon plasma is shown in Fig. 3a, and in Fig. 3b the thicknesses of cathode glow and Crookes dark space regions in the tube at different argon gas pressures are plotted. We had almost a violet emission from argon plasma which was not changed after adding $20 \%$ oxygen to the working gas. As is clear from the figure, several discharge regions which are introduced classically in the texts could be observed during the discharge. Cathode surface was always luminous due to high-energy ion impacts, and immediately in front of the cathode the cathode glow region can be observed. This region is a thin bright region of the order of less than $1 \mathrm{~cm}$ thickness which corresponds to excited atoms sputtered off the cathode surface, or incoming positive ions which are moving toward the cathode. Since this region consists of high ion number density its thickness is changed with change in the pressure of the gas during the discharge. After cathode glow region a dark region can be observed in 

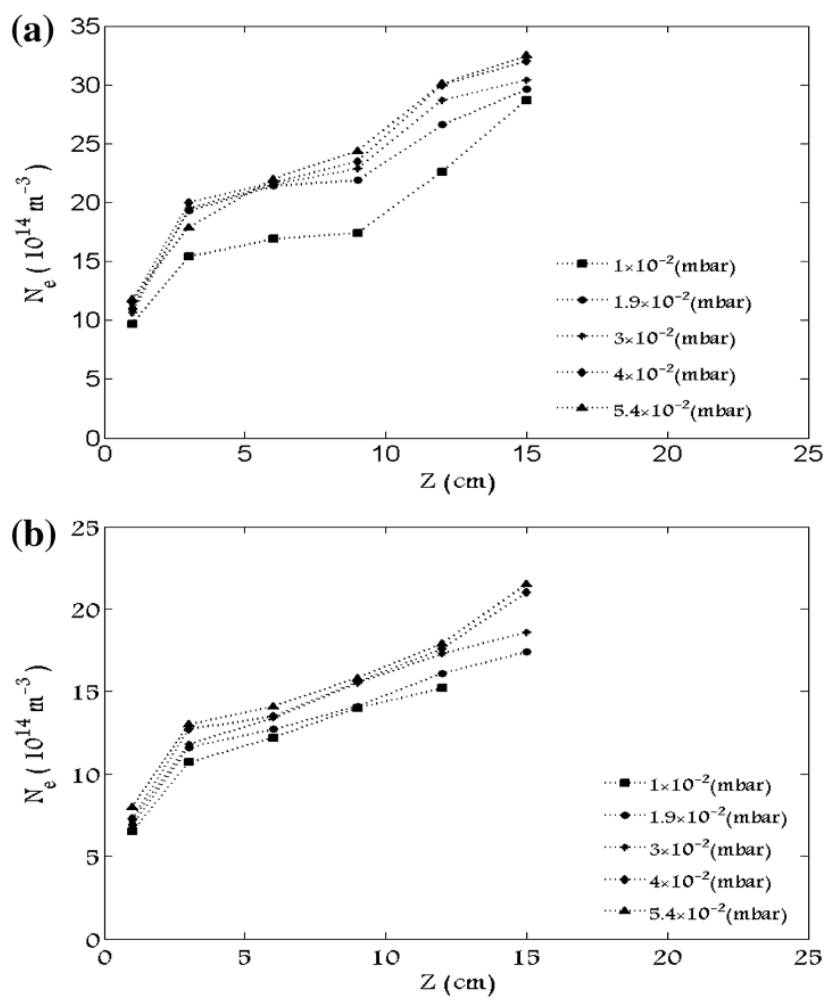

Fig. 4 Plasma electron density at different points on the axis of discharge tube at different pressures for $\mathrm{Ar}$ plasma (a) and $\mathrm{Ar} / \mathrm{O}_{2}$ plasma (b)

Fig. 3a. This region is the known as Crookes dark space. And immediately after this dark band one can see the brightest light intensity in the entire discharge tube which is the region of negative glow. Electrons which have been accelerated in the cathode region produce ionization and intense excitation in this region. At any pressure, we could not observe Faraday dark space in the discharge tube. In fact in this regime of electrical discharge after negative glow region electrons achieve enough energy in a short time to make ionization and excitation of neutrals to start positive column in the discharge tube in contact with negative glow region. Positive column is elongated to anode dark space. The thickness of cathode glow region depends on the type of gas and the gas pressure [1]. As is clear in Fig. 3b, thicknesses of both Crookes dark space and cathode glow regions are decreased with increase in the pressure while the thickness of positive column is increased.

Because of the thickness of the LP tube, we could not do any measurement in the anode dark and cathode glow regions, and in the Crookes dark space the current of the probe was zero for the interval -50 to $+50 \mathrm{~V}$.

In Fig. 4a and b, variation of plasma electron density along the discharge tube at different discharge pressures for $\mathrm{Ar}$ and $\mathrm{Ar} / \mathrm{O}_{2}$ plasmas are presented. And in Fig. 5a and b,
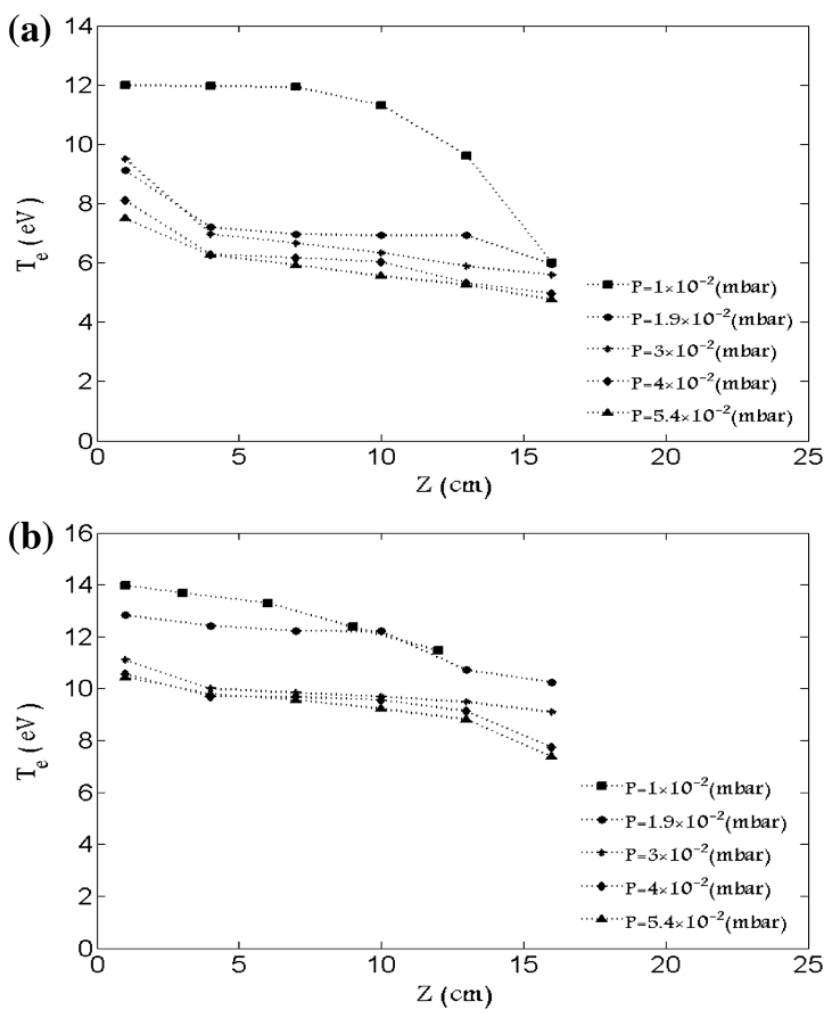

Fig. 5 Plasma electron temperature at different points on the axis of discharge tube at different pressures for $\mathrm{Ar}$ plasma (a) and $\mathrm{Ar} / \mathrm{O}_{2}$ plasma (b)

the plasma electron temperature at these points can be observed. From cathode to anode, the density of electrons is decreased while their temperature is increased. With drifting from cathode to anode, electrons achieve energy from applied electric field which leads to increase their temperature. By increasing the temperature $v_{\mathrm{ei}}$ the collision frequency which is inversely proportional to electron temperature is decreased. Smaller number of ionizing collision decreases the electron densities. After introducing oxygen as an electronegative gas, the electron density is decreased while the electron temperature is increased as compared to pure Ar plasma. In fact number of ionizing collisions is decreased. Plasma current is kept constant in both $\mathrm{Ar}$ and $\mathrm{Ar} / \mathrm{O}_{2}$ plasmas. But in $\mathrm{Ar} / \mathrm{O}_{2}$ plasma some electrons are bonded to oxygen atoms to make negative ions. Because plasma is quasineutral, the number of free electrons in $\mathrm{Ar} / \mathrm{O}_{2}$ plasma is less than $\mathrm{Ar}$ plasma. Decreasing the electron density leads to decreasing the collision numbers so in the case of $\mathrm{Ar} / \mathrm{O}_{2}$ plasma electron energy is higher.

Variation of the floating potential in the discharge tube for both cases of argon and argon-oxygen plasmas is shown in Fig. 6a and b. Floating potential is the potential at which current of LP is zero. In this experiment, the measured floating potential is increased from cathode to anode. 

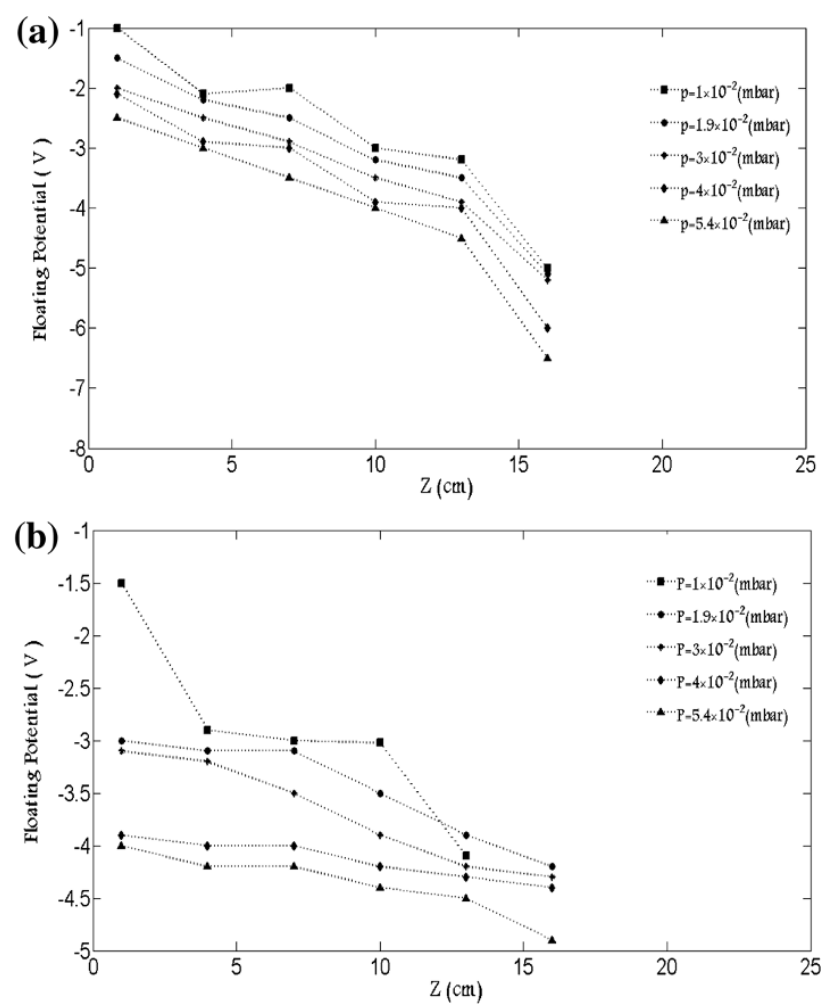

Fig. 6 Floating potential at different points on the axis of discharge tube at different pressures for $\mathrm{Ar}$ plasma (a) and $\mathrm{Ar} / \mathrm{O}_{2}$ plasma (b)

Increasing the floating potential shows an increase in the number of electrons in the Debye sphere. Number of electrons in the Debye sphere is proportional to electron temperature and inversely proportional to electron density with different powers as [16]

$N_{\mathrm{D}} \sim T^{3 / 2} / n_{0}^{1 / 2}$

where $N_{\mathrm{D}}$ is the number of electrons in the Debye sphere and $T$ and $n_{0}$ are the plasma electron density and temperature. As is shown in Figs. 4 and 5, in the discharge tube electron temperature was increased from cathode to anode while electron density was decreased. Consequently, floating potential is increased from cathode to anode. In the case of argon-oxygen plasma, we have less number of electrons in the Debye sphere so smaller probe voltage is required to stop the probe current.

Variation of plasma potential $V_{\mathrm{p}}$ on the axis of discharge tube is presented in Fig. 7a and $b$ for argon and argonoxygen plasmas, respectively. The plasma potential is the potential of the plasma with respect to the walls of the device at a given location in plasma. Generally, the walls of discharge tube are at negative potential due to runaway electrons of plasma. So $V_{\mathrm{p}}$ is generally a few volts positive with respect to the walls [17]. The plasma potential is inferred from the intersection of two tangent lines to the curve $\ln \left(I_{\text {probe }}\right)$ verses $V_{\text {probe }}$ for $V_{\text {probe }}>V_{\mathrm{p}}$ and
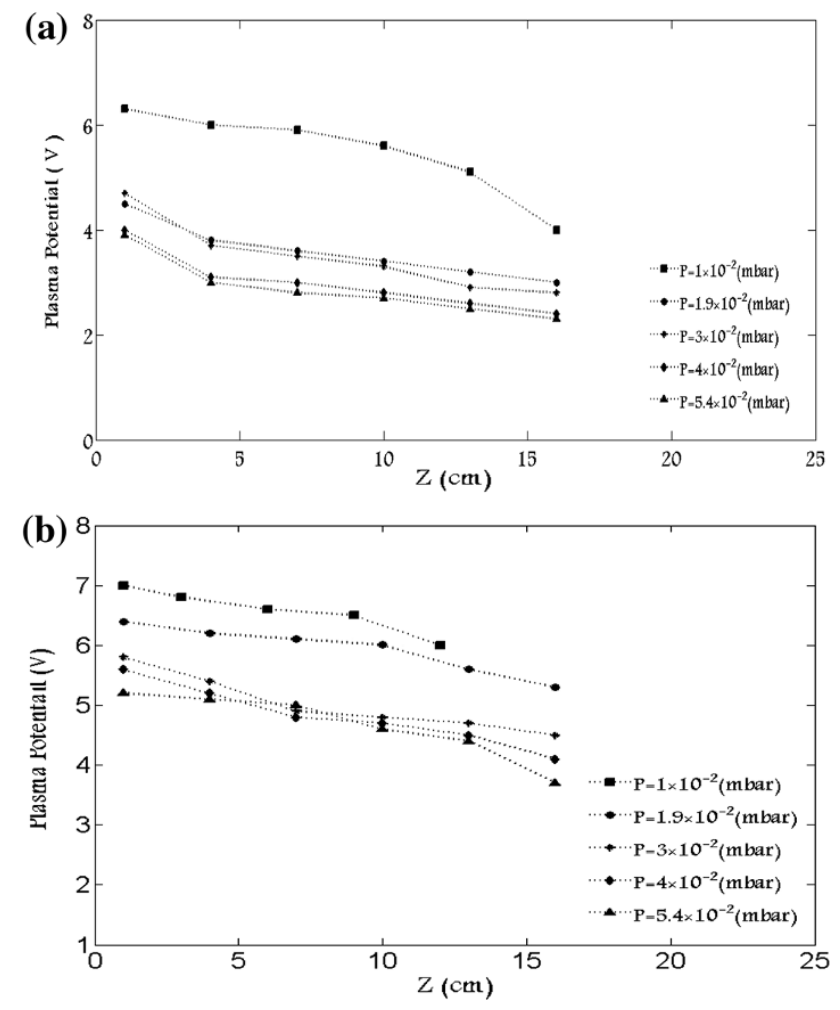

Fig. 7 Plasma potential at different points on the axis of discharge tube at different pressures for Ar plasma (a) and $\mathrm{Ar} / \mathrm{O}_{2}$ plasma (b)

$V_{\text {probe }}<V_{\mathrm{p}}$ [18]. The plasma potential of both argon and argon-oxygen plasmas is increased on the axis of discharge tube from cathode to anode as is shown in Fig. 7. The reason can be due to decreasing the electron density from cathode to anode as is shown in Fig. 4. In the case of argon oxygen plasma, electron density of the axis is smaller so we have a larger plasma potential on the axis of discharge tube.

\section{Conclusion}

Glow discharge of both pure argon and argon-oxygen plasmas has been studied at different pressures and constant current mode equal to $10 \mathrm{~mA}$. Results show that increasing the working pressure gives rise to increase in the number of carriers of electrical current (electrons and ions) and collision frequency. But increasing the collision frequency is not as effective as increasing the number of current carriers on the discharge voltage so a lower discharge voltage is required to sustain the discharge process.

We have investigated changes of plasma parameters from cathode to anode on the axis of discharge tube at different pressures and constant current mode. It is found that for both cases of pure argon and argon-oxygen plasmas, the electron temperature is increased from cathode to 
anode while the electron density is decreased. Plasma potential and floating potential are increased from cathode to anode. On the other hand, increasing the discharge pressure leads to reduction in the plasma electron temperature. The electronegativity of oxygen decrease the electron density which leads to decrease the floating potential and increase the plasma potential.

Open Access This article is distributed under the terms of the Creative Commons Attribution License which permits any use, distribution, and reproduction in any medium, provided the original author(s) and the source are credited.

\section{References}

1. Fridman, A.: Plasma Chemistry. Cambridge University Press, Cambridge (2008)

2. Joh, H.M., Chung, T.H., Chung, K.-S.: Thin Solid Films 518, 6686-6689 (2010)

3. Stoffels, E., Stoffels, W.W., Vender, D., Kando, M., Kroesen, G.M.W., de Hoog, F.J.: Phys. Rev. E 51, 2425 (1995)

4. Lichtenberg, A.J., Lieberman, M.A., Kouznetsov, I.G., Chung, T.H.: Plasma Sources Sci. Technol. 9, 45 (2000)
5. Quandt, E., Fobele, H.F., Graham, W.G.: Appl. Phys. Lett. 72, 2394 (1998)

6. Chung, K.-S., Kado, S.: Phys. Plasmas 13, 104509 (2006)

7. Godyak, V.A., Piejak, R.B., Alexandrovich, B.A.: Plasma Sources Sci. Technol. 1, 36 (1992)

8. Vucelic, M., Mijovic, S.: J. Appl. Phys. 84, 4731 (1998)

9. Hayashi, D., Kadota, K.: J. Appl. Phys. 83, 697 (1998)

10. Noguchi, M., Hirao, T., Shindo, M., Sakurauchi, K., Yamagata, Y., Uchino, K., Kawai, Y., Muraoka, K.: Plasma Sources Sci. Technol. 12, 403 (2003)

11. Roth, J.R.: Industrial Plasma Engineering. IOP Publishing Ltd, Bristol (1995)

12. Yasserian, K., Ghornneviss, M., Aslaninejad, M.: Jpn. J. Appl. Phys. 48, 036001 (2009)

13. Araghi, F., Dorranian, D.: JTAP 7, 41 (2013)

14. Passoth, E., Kudrna, P., Csambal, C., Behnke, J.F., Tichy, M., Helbig, V.: J. Phys. D 30, 1763 (1997)

15. Noguchi, M., Hirao, T., Sakurauchi, K., Yamagata, Y., Uchino, K., Kawai, Y., Muraoka, K.: Plasma Sources Sci. Technol. 12, 403 (2003)

16. Huddlestone, R.H., Leonard, S.L. (eds.): Plasma Diagnostic Techniques. Academic Press (1965)

17. Robert, L.M.: Understanding Langmuir probe current-voltage characteristics. Am. J. Phys. 75(12), 1078-1085 (2007)

18. Hung, S., Ning, Z.Y., Xin, Y., Di, X.L.: Surf. Coat. Technol. 200, 3963-3968 (2006) 